ALEA, Lat. Am. J. Probab. Math. Stat. 15, 691-702 (2018)

DOI: 10.30757/ALEA.v15-26

\title{
Central Limit Theorem for one and two dimensional Self-Repelling Diffusions
}

\section{Carl-Erik Gauthier}

University of Washington

Department of Mathematics, Box 354350,

Seattle, WA 98195, USA.

E-mail address: carlgaut@uw.edu

Abstract. We prove a Central Limit Theorem for the finite dimensional distributions of the displacement for the 1-dimensional self-repelling diffusion which solves

$$
d X_{t}=d B_{t}-\left(G^{\prime}\left(X_{t}\right)+\int_{0}^{t} F^{\prime}\left(X_{t}-X_{s}\right) d s\right) d t,
$$

where $B$ is a real valued standard Brownian motion, $G$ is an initial environment and $F(x)=\sum_{k=1}^{n} a_{k} \cos (k x)$ with $n<\infty$ and $a_{1}, \cdots, a_{n}>0$. A 2-dimensional extension is also discussed.

In dimension $d \geq 3$, such a result has already been established by Horváth, Tóth and Vetö in 2012 for a large class of interaction functions $F$, but not for $d=1,2$. Under an integrability condition, Tarrès, Tóth and Valkó conjectured that a Central Limit Theorem result should also hold in dimension $d=1$.

\section{Introduction}

In this short note, our main goal is to prove the Central Limit Theorem (denoted by CLT in the sequel) for the finite dimensional distribution of the displacement for the one-dimensional self-repelling diffusion solving

$$
d X_{t}=d B_{t}-\left(G^{\prime}\left(X_{t}\right)+\int_{0}^{t} F^{\prime}\left(X_{t}-X_{s}\right) d s\right) d t, \quad X_{0}=0,
$$

where $B$ is a real valued standard Brownian motion, $G(x)=\sum_{k=1}^{n}\left(u_{k} \cos (k x)+\right.$ $\left.v_{k} \sin (k x)\right)$ and $F(x)=\sum_{k=1}^{n} a_{k} \cos (k x)$ with $n<\infty$ and $a_{1}, \cdots, a_{n}>0$. The function $G$ provides the initial environment of the particle and $F$ is the interaction function.

Received by the editors October 26th, 2017; accepted May 9th, 2018.

2010 Mathematics Subject Classification. 60F05, 60K35, 60H10, 60J60.

Key words and phrases. Central Limit Theorem, Self-Repelling Diffusion.

Research supported by the Swiss National Foundation for Research, grants 200021_163072 and P2NEP2_171951. 
Roughly speaking, self-repelling diffusions (as considered here) are time continuous stochastic processes which solve an inhomogeneous stochastic differential equation whose drift part is evolving in time according to the whole past history of the process in such a way that it tends to push the diffusing particle away from the most visited sites.

Under the assumptions made on $F$ and $G$, the Law of Large Number has already been established in Benaïm and Gauthier (2017, Theorem 2 and Remark 1), namely

$$
\lim _{t \rightarrow \infty} \frac{X_{t}}{t}=0 \text { a.s. }
$$

A question that one may then ask is whether or not a CLT result holds. The purpose of this note is to provide a positive answer to it (see Theorem 2.1).

The question whether or not a CLT result could be established for self-repelling diffusions was first investigated in 2012 by Tarrès, Tóth and Valkó (Tarrès et al., 2012, Theorem 2 and its remark) and shortly later Horváth, Tóth and Vetö were able to prove in 2012 a full CLT result (Horváth et al., 2012, Theorem 2) in dimensions $d \geq 3$. In both papers, the authors consider a self-repelling diffusion that solves

$$
d X_{t}=d B_{t}-\left(\nabla G\left(X_{t}\right)+\int_{0}^{t} \nabla F\left(X_{t}-X_{s}\right) d s\right) d t,
$$

where $F: \mathbb{R}^{d} \rightarrow \mathbb{R}$ is a smooth spherically symmetric function with non-negative Fourier transform and some additional technical assumptions that be found in the respective papers.

Under those conditions, they proved that the process $t \mapsto \eta_{t}$ defined by

$$
\eta_{t}(x)=\nabla G\left(x+X_{t}\right)+\int_{0}^{t} \nabla F\left(x+X_{t}-X_{s}\right) d s
$$

for $x \in \mathbb{R}^{d}$ is a Markov process with almost-surely continuous sample path in a suitable chosen infinite dimensional function space and admits a Gaussian distribution as stationary and ergodic distribution. This allowed the authors to prove that a Law of Large Numbers for $X_{t}$ holds.

The authors were also able to prove from the so-called Yaglom-reversibility and $\mathcal{H}_{-1}$ estimates that

$$
d \leq \liminf _{t \rightarrow \infty} \mathbb{E}\left(\left|X_{t}\right|^{2}\right) \leq \limsup _{t \rightarrow \infty} \mathbb{E}\left(\left|X_{t}\right|^{2}\right) \leq d \int_{\mathbb{R}^{d}} \frac{\hat{F}(p)}{|p|^{2}} d p .
$$

When

$$
\int_{\mathbb{R}^{d}} \frac{\hat{F}(p)}{|p|^{2}} d p<\infty
$$

a diffusive scaling, and eventually a CLT for $X_{t} / \sqrt{t}$ is naturally expected. In dimension $d \geq 3$, Horváth, Tóth and Vetö proved in Horváth et al. (2012) that $\liminf _{t \rightarrow \infty} \mathbb{E}\left(\left|X_{t}\right|^{2}\right)=\limsup _{t \rightarrow \infty} \mathbb{E}\left(\left|X_{t}\right|^{2}\right)$ and established the full CLT by checking that the graded sector conditions held. This allowed them to use the KipnisVaradhan's CLT result for additive functionals.

Due to technical obstructions, they were not able to extend lower dimensions. Therefore, in dimension $d=1,2$, the question of a CLT result remained open. Theorem 2.1 below fills the gap for particular 1-dimensional cases, whereas Theorem 3.1 fills the gap for particular 2-dimensional cases. 
Before turning to the presentation of the result in dimension one, let us briefly make a link between the positiveness condition of the Fourier transform of $F$ and the positiveness of the coefficients $a_{1}, \cdots, a_{n}$. Let $b \in L^{1}(\mathbb{R}) \cap C^{\infty}(\mathbb{R})$ be a fast decaying function ${ }^{1}$ such that its Fourier transform is non-negative and let

$$
\varphi_{2 \pi}(b)(x)=\sum_{n=-\infty}^{\infty} b(x+2 \pi n)
$$

be the $2 \pi$-periodization transform of $b$. It is an exercise in Fourier analysis to show that

$$
\varphi_{2 \pi}(b)(x)=\frac{1}{2 \pi} \sum_{k \in \mathbb{Z}} \hat{b}\left(\frac{k}{2 \pi}\right) e^{i k x} .
$$

Because $b$ is even, we have

$$
\varphi_{2 \pi}(b)(x)=\frac{\hat{b}(0)}{2 \pi}+\frac{1}{\pi} \sum_{k \geqslant 1} \hat{b}\left(\frac{k}{2 \pi}\right) \cos (k x) .
$$

The paper is organized as follows. In Section 2, we state the CLT result and present the tools and concepts involved for the one-dimensional case and end the section with the proof of the CLT. The two-dimensional case will be discussed in Section 3. Because straightforward adaptations of the 1-dimensional case (but with more cumbersome computations) gives the 2-dimensional case once the right change of variable is made, we will only present the changes of variable that need to done in order to be in the same framework as for the one dimensional case.

\section{The one-dimensional case}

The purpose of this section is to prove the following.

\section{Theorem 2.1.}

Let $\left(X_{t}\right)_{t}$ be the solution of (1.1). Then:

(1) $\sigma^{2}:=\lim _{t \rightarrow \infty} \frac{\mathbb{E}_{\pi}\left(X_{t}^{2}\right)}{t}$ exists and it satisfies

$$
1 \leq \sigma^{2} \leq 1+2\left(\sum_{j=1}^{n} \frac{a_{j}}{j^{2}}\right)
$$

(2) For any $0<t_{1}<\cdots<t_{n}<\infty$, we have

$$
\left(\frac{\sqrt{\varepsilon} X_{t_{1} / \varepsilon}}{\sigma}, \cdots, \frac{\sqrt{\varepsilon} X_{t_{n} / \varepsilon}}{\sigma}\right) \stackrel{\varepsilon \rightarrow 0}{\Longrightarrow}\left(W_{t_{1}}, \cdots, W_{t_{n}}\right)
$$

under $\mathbb{P}_{\pi}$, where $W$ is a real valued standard Brownian motion. Here $\pi$ is the probability measure over $\mathbb{R}^{2 n}$ defined by (2.7) and $\Longrightarrow$ denotes the convergence in distribution.

Following the same idea as in Benaïm and Gauthier (2017), set $U_{j}(t)=u_{j}+$ $\int_{0}^{t} \cos \left(j X_{s}\right) d s$ and $V_{j}(t)=v_{j}+\int_{0}^{t} \sin \left(j X_{s}\right) d s$. With these new variables, we obtain

\footnotetext{
${ }^{1} \mathrm{~A}$ function $b$ is said fast decaying if for any $k \geq 0$, there exists $c_{k}>0$ such that for any $x \in \mathbb{R}$, $|b(x)| \leq c_{k} /\left(1+|x|^{k}\right)$.
} 
the following system

$$
\left\{\begin{array}{l}
d X_{t}=d B_{t}+\sum_{j=1}^{n} j a_{j}\left(\sin \left(j X_{t}\right) U_{j}(t)-\cos \left(j X_{t}\right) V_{j}(t)\right) d t \\
d U_{j}(t)=\cos \left(j X_{t}\right) d t, j=1, \ldots, n . \\
d V_{j}(t)=\sin \left(j X_{t}\right) d t, j=1, \ldots, n .
\end{array}\right.
$$

Since for all $j=1 \cdots, n$, the functions $x \mapsto \cos (j x)$ and $x \mapsto \sin (j x)$ are $2 \pi$ periodic, we can replace $X_{t}$ by $\Theta_{t}=X_{t}(\bmod 2 \pi) \in S^{1}$, where $S^{1}=\mathbb{R} / 2 \pi \mathbb{Z}$ denotes the 1-dimensional flat torus. This replacement allows us to use the framework from Benaïm and Gauthier (2017).

In order to shorten the notation, we let $U(t)$ and $V(t)$ denote the vectors

$$
U(t)=\left(U_{1}(t), \cdots, U_{n}(t)\right) \quad \text { and } \quad V(t)=\left(V_{1}(t), \cdots, V_{n}(t)\right) .
$$

Summarizing the main results from Benaïm and Gauthier (2017), we have

Theorem 2.2 (Theorem 5 and Theorem 6, Benaïm and Gauthier, 2017). Let $\left(P_{t}\right)_{t \geqslant 0}$ be the semi-group associated to the process $\left(\left(\Theta_{t}, U(t), V(t)\right)\right)_{t \geq 0}$ and denote by $P_{t}\left(\left(\theta_{0}, U(0), V(0)\right), d \theta d u d v\right)$ its transition probability. Then

1) The unique invariant probability measure is

$$
\mu(d \theta d u d v)=\nu(d \theta) \otimes \frac{e^{-\Phi(u, v)}}{C} d u d v
$$

where $\Phi(u, v)=\frac{1}{2} \sum_{k=1}^{n} a_{k} k^{2}\left(u_{k}^{2}+v_{k}^{2}\right), C$ is a normalization constant and $\nu(d \theta)$ is the uniform probability measure on $S^{1}=\mathbb{R} / 2 \pi \mathbb{Z}$.

2) Let $\mu_{t}=\mathcal{L}\left(\Theta_{t}, U(t), V(t)\right)$ denote the law of $\left(\Theta_{t}, U(t), V(t)\right)$. Then for any initial distribution $\mu_{0}, \mu_{t}$ converges to $\mu$ in total variation.

3) For every $\eta>0$ and $g \in L^{2}(\mu)$

$$
\left\|P_{t} g-\int g(\theta, u, v) \mu(d \theta d u d v)\right\|_{L^{2}(\mu)} \leqslant \sqrt{1+2 \eta}\left\|g-\int g(\theta, u, v) \mu(d \theta d u d v)\right\|_{L^{2}(\mu)} e^{-\lambda t},
$$

where

$$
\lambda=\frac{\eta}{1+\eta} \frac{K_{1}}{1+K_{2}+K_{3}},
$$

with explicit constants $K_{1}, K_{2}$ and $K_{3}$.

In this paper, we will adopt the same point of view as in Tarrès et al. (2012): the environment seen from the particle. For that purpose, introduce the following new variables

$$
C_{j}(t)=U_{j}(t) \cos \left(j X_{t}\right)+V_{j}(t) \sin \left(j X_{t}\right)=\left\langle\left(\begin{array}{c}
U_{j}(t) \\
V_{j}(t)
\end{array}\right),\left(\begin{array}{c}
\cos \left(j X_{t}\right) \\
\sin \left(j X_{t}\right)
\end{array}\right)\right\rangle
$$

and

$$
S_{j}(t)=\sin \left(j X_{t}\right) U_{j}(t)-\cos \left(j X_{t}\right) V_{j}(t)=\left\langle\left(\begin{array}{c}
U_{j}(t) \\
V_{j}(t)
\end{array}\right),\left(\begin{array}{c}
\sin \left(j X_{t}\right) \\
-\cos \left(j X_{t}\right)
\end{array}\right)\right\rangle .
$$

So, if we denote by $\eta_{t}$ the potential viewed from the particle's position, i.e

$$
\eta_{t}(x)=\int_{0}^{t} F\left(x+X_{t}-X_{s}\right) d s+G\left(x+X_{t}\right)
$$


then

$$
\eta_{t}(x)=\sum_{k=1}^{n} a_{k}\left(C_{k}(t) \cos (k x)-S_{k}(t) \sin (k x)\right) .
$$

Moreover, this allows us to rewrite $X_{t}$ as

$$
X_{t}=B_{t}+\int_{0}^{t} \sum_{k=1}^{n} k a_{k} S_{k}(u) d u=B_{t}-\int_{0}^{t} \varphi\left(\eta_{u}^{\prime}\right) d u
$$

where $\varphi: \Omega \rightarrow \mathbb{R}$ is defined by $\varphi(\omega)=\omega(0)$ and $\Omega$ is the vector space spanned by the functions $\cos (k x)$ and $\sin (k x)$ for $k=0,1, \cdots n$.

Before diving into the technical results, let us introduce the following notation. We denote by $T_{t}$ the semigroup induced by the process

$$
((C(t), S(t)))_{t \geqslant 0}:=\left(\left(C_{1}(t), S_{1}(t), \cdots, C_{n}(t), S_{n}(t)\right)\right)_{t \geqslant 0}
$$

and by $G$ its infinitesimal generator. For an operator $R$, we denote its domain by $D(R)$.

Given a probability measure $\pi$ over $\mathbb{R}^{2 n}$, we denote by $L^{2}(\pi)$ the space $L^{2}\left(\mathbb{R}^{2 n}, \pi\right)$, by $\langle., .\rangle_{L^{2}(\pi)}$ the associated inner product and by $\|\cdot\|_{L^{2}(\pi)}$ the induced $L^{2}-$ norm.

The dynamic of $((C(t), S(t)))_{t \geqslant 0}$ is described by applying Itô's formula to $(2.4)$ and (2.5), which yields

$$
d\left(\begin{array}{c}
C_{1}(t) \\
S_{1}(t) \\
C_{2}(t) \\
S_{2}(t) \\
\vdots \\
C_{n}(t) \\
S_{n}(t)
\end{array}\right)=\left(\begin{array}{c}
-S_{1}(t) \\
C_{1}(t) \\
-2 S_{2}(t) \\
2 C_{2}(t) \\
\vdots \\
-n S_{n}(t) \\
n C_{n}(t)
\end{array}\right)\left(d B_{t}+\left(\sum_{k=1}^{n} k a_{k} S_{k}(t)\right) d t\right)-\frac{1}{2}\left(\begin{array}{c}
C_{1}(t) \\
S_{1}(t) \\
4 C_{2}(t) \\
4 S_{2}(t) \\
\vdots \\
n^{2} C_{n}(t) \\
n^{2} S_{n}(t)
\end{array}\right) d t+\left(\begin{array}{c}
1 \\
0 \\
1 \\
0 \\
\vdots \\
1 \\
0
\end{array}\right) d t .
$$

\section{Proposition 2.3.}

(1) For any smooth function $f$ having compact support, we have

$$
\begin{aligned}
G f(c, s)= & \frac{1}{2} \sum_{j=1}^{n} j^{2}\left(s_{j}^{2} \partial_{c_{j} c_{j}} f+c_{j}^{2} \partial_{s_{j} s_{j}} f\right)-\sum_{j=1}^{n} j^{2} s_{j} c_{j} \partial_{c_{j} s_{j}} f \\
& +\frac{1}{2} \sum_{k \neq j} j k\left(s_{j} s_{k} \partial_{c_{k} c_{j}} f+c_{j} c_{k} \partial_{s_{j} s_{k}} f\right)-\sum_{k \neq j}^{n} j k s_{j} c_{k} \partial_{c_{j} s_{k}} f \\
& +\left(\sum_{k=1}^{n} k a_{k} s_{k}\right) \sum_{j=1}^{n} j\left(-s_{j} \partial_{c_{j}} f+c_{j} \partial_{s_{j}} f\right) \\
& -\frac{1}{2} \sum_{j=1}^{n} j^{2}\left(c_{j} \partial_{c_{j}} f+s_{j} \partial_{s_{j}} f\right)+\sum_{j=1}^{n} \partial_{c_{j}} f .
\end{aligned}
$$

(2) The process $((C(t), S(t)))_{t \geqslant 0}$ admits a unique invariant probability measure of the form

$$
\pi(d c d s)=\frac{e^{-\Phi(c, s)}}{C} d c d s
$$


where $\Phi(c, s)=\frac{1}{2} \sum_{k=1}^{n} a_{k} k^{2}\left(c_{k}^{2}+s_{k}^{2}\right)$ and $C$ is the normalizing constant.

(3) For any function $f \in L^{2}(\pi)$, we have

$$
\left\|T_{t} f-\int_{\mathbb{R}^{2 n}} f(c, s) \pi(d c d s)\right\|_{L^{2}(\pi)} \leq \sqrt{3}\left\|f-\int_{\mathbb{R}^{2 n}} f(c, s) \pi(d c d s)\right\|_{L^{2}(\pi)} e^{-\lambda t},
$$

where $\lambda=\frac{1}{2} \frac{K_{1}}{1+K_{2}+K_{3}}$ and the constants $K_{1}, K_{2}$ and $K_{3}$ are those from Theorem 2.2.

Proof:

(1) The result follows from (2.6), Itô's formula and Revuz and Yor (1999, Propositions VII.1.6 and VII.1.7).

(2) The fact that $\pi(d c d s)$ is an invariant probability measure follows from Theorem 2.2 as well as from the equations (2.4) and (2.5). Indeed, for any $A_{j} \in \mathcal{B}\left(\mathbb{R}^{2}\right)$, we have by rotation invariance of the Gaussian measure

$$
\begin{aligned}
\pi\left(A_{1} \times \cdots \times A_{n}\right) & =\mathbb{P}\left(\left(C_{j}(t), S_{j}(t)\right) \in A_{j} \forall j=1, \cdots, n\right) \\
& =\mathbb{P}\left(\left(U_{j}(t), V_{j}(t)\right) \in A_{j} \forall j=1, \cdots, n\right) \\
& =\mathbb{P}\left(\Theta_{t} \in \mathbb{S}^{1},\left(U_{j}(t), V_{j}(t)\right) \in A_{j} \forall j=1, \cdots, n\right) \\
& =\mu\left(\mathbb{S}^{1} \times A_{1} \times \cdots \times A_{n}\right)
\end{aligned}
$$

Concerning the uniqueness, let $\nu$ be an invariant probability measure for the process $((C(t), S(t)))_{t \geqslant 0}$. Then define on $S^{1} \times \mathbb{R}^{n} \times \mathbb{R}^{n}$ the probability measure $\mu_{0}(d \theta d u d v)=\delta_{0} \otimes \nu(d u d v)$ and sample $\left(\Theta_{0}, U(0), V(0)\right)$ according to $\mu_{0}$.

By Theorem 2.2, $\mu_{t}$ converges to $\mu$ in total variation. In particular the marginal law of $\mu_{t}$ corresponding to $(U(t), V(t))$ converges to $\pi$. Thus $\nu=\pi$.

(3) Let $f: \mathbb{R}^{n} \times \mathbb{R}^{n} \rightarrow \mathbb{R}$ and define a function $g: S^{1} \times \mathbb{R}^{n} \times \mathbb{R}^{n} \rightarrow \mathbb{R}$ by

$$
g(\theta, u, v)=f(c, s),
$$

where the pairs $\left(c_{j}, s_{j}\right)$ are defined as in (2.4) and (2.5). Since the evolution of $(C(t), S(t))$ does not depend on the dynamic of $\Theta_{t}$, we have

$$
\begin{aligned}
T_{t} f(c, s) & =\mathbb{E}(f(C(t), S(t)) \mid C(0)=c, S(0)=s) \\
& =\mathbb{E}\left(f(C(t), S(t)) \mid \Theta_{0}=\theta, C(0)=c, S(0)=s\right) \\
& =\mathbb{E}\left(f(C(t), S(t)) \mid \Theta_{0}=\theta, U(0)=u, V(0)=v\right) \\
& =\mathbb{E}\left(g\left(\Theta_{t}, U(t), V(t)\right) \mid \Theta_{0}=\theta, U(0)=u, V(0)=v\right) \\
& =P_{t} g(\theta, u, v)
\end{aligned}
$$

where the pairs $\left(u_{k}, v_{k}\right)$ are such that $c_{k}=u_{k} \cos (k \theta)+v_{k} \sin (k \theta)$ and $s_{k}=u_{k} \sin (k \theta)-v_{k} \cos (k \theta)$.

The statement follows then from Theorem 2.2 with $\eta=1$. 
Proposition 2.4. Let $K, A$ be the operators defined on smooth functions $f$ with compact support by

$$
\begin{aligned}
K f(c, s)= & \frac{1}{2} \sum_{j=1}^{n} j^{2}\left(s_{j}^{2} \partial_{c_{j} c_{j}} f+c_{j}^{2} \partial_{s_{j} s_{j}} f\right)-\sum_{j=1}^{n} j^{2} s_{j} c_{j} \partial_{c_{j} s_{j}} f \\
& +\frac{1}{2} \sum_{k \neq j} j k\left(s_{j} s_{k} \partial_{c_{k} c_{j}} f+c_{j} c_{k} \partial_{s_{j} s_{k}} f\right)-\sum_{k \neq j}^{n} j k s_{j} c_{k} \partial_{c_{j} s_{k}} f \\
& -\frac{1}{2} \sum_{j=1}^{n} j^{2}\left(c_{j} \partial_{c_{j}} f+s_{j} \partial_{s_{j}} f\right)
\end{aligned}
$$

and

$$
A f(c, s)=\left(\sum_{k=1}^{n} k a_{k} s_{k}\right) \sum_{j=1}^{n} j\left(-s_{j} \partial_{c_{j}} f+c_{j} \partial_{s_{j}} f\right)+\sum_{j=1}^{n} \partial_{c_{j}} f .
$$

Then $K$ is symmetric over $L^{2}(\pi)$, while the operator $A$ is skew-symmetric.

Proof: Let $j \in\{1, \cdots, n\}$ and let $f, g$ be smooth functions with compact support. Then, integrations by parts yield

$$
\begin{aligned}
& \frac{1}{2} \int j^{2}\left(s_{j}^{2} \partial_{c_{j} c_{j}} f+c_{j}^{2} \partial_{s_{j} s_{j}} f\right) g d \pi-\int j^{2} s_{j} c_{j} \partial_{c_{j} s_{j}} f g d \pi \\
& =\frac{1}{2} \int j^{2}\left(s_{j}^{2} \partial_{c_{j} c_{j}} f+c_{j}^{2} \partial_{s_{j} s_{j}} f\right) g d \pi-\int j^{2} s_{j} c_{j} \partial_{c_{j} s_{j}} f g d \pi \\
& +\frac{1}{2} \int j^{4} a_{j}\left(c_{j}^{2}+s_{j}^{2}\right) f g d \pi-\int j^{2}\left(c_{j} \partial_{c_{j}} g+s_{j} \partial_{s_{j}} g\right) f d \pi-\int j^{2} f g d \pi \\
& \frac{1}{2} \int j^{2}\left(c_{j} \partial_{c_{j}} f+s_{j} \partial_{s_{j}} f\right) g d \pi=-\frac{1}{2} \int j^{2}\left(c_{j} \partial_{c_{j}} g+s_{j} \partial_{s_{j}} g\right) f d \pi-\int j^{2} f g d \pi \\
& +\frac{1}{2} \int j^{4} a_{j}^{2}\left(c_{j}^{2}+s_{j}^{2}\right) f g d \pi \\
& \int \partial_{c_{j}} f g d \pi=-\int \partial_{c_{j}} g f d \pi+\int j^{2} a_{j} c_{j} f g d \pi
\end{aligned}
$$

and

$$
\begin{aligned}
\int j^{2} a_{j}\left(-s_{j}^{2} \partial_{c_{j}} f+s_{j} c_{j} \partial_{s_{j}} f\right) g d \pi= & -\int j^{2} a_{j}\left(-s_{j}^{2} \partial_{c_{j}} g+s_{j} c_{j} \partial_{s_{j}} g\right) f d \pi \\
& -\int a_{j} j^{2} c_{j} f g d \pi
\end{aligned}
$$

For $k \neq j$, we have

$$
\int j k a_{k}\left(-s_{j} s_{k} \partial_{c_{j}} f+s_{k} c_{j} \partial_{s_{j}} f\right) g d \pi=-\int j^{2} a_{j}\left(-s_{j}^{2} \partial_{c_{j}} g+s_{j} c_{j} \partial_{s_{j}} g\right) f d \pi
$$


and

$$
\begin{aligned}
& \frac{1}{2} \int j k\left(s_{j} s_{k} \partial_{c_{j} c_{k}} f+c_{j} c_{k} \partial_{s_{j} s_{k}} f\right) g d \pi-\int j k s_{j} c_{k} \partial_{c_{j} s_{k}} f g d \pi \\
&=\frac{1}{2} \int j k\left(s_{j} s_{k} \partial_{c_{j} c_{k}} f+c_{j} c_{k} \partial_{s_{j} s_{k}} g\right) f d \pi-\int j k s_{j} c_{k} \partial_{c_{j} s_{k}} f g d \pi \\
& \quad+\frac{1}{2} \int j k^{3} a_{k} c_{k} s_{j} s_{k} \partial_{c_{j}} g f d \pi-\frac{1}{2} \int k j^{3} a_{j} c_{j} s_{k} s_{j} \partial_{c_{k}} g f d \pi \\
& \quad+\frac{1}{2} \int k j^{3} a_{j} s_{j} c_{k} c_{j} \partial_{s_{k}} g f d \pi-\frac{1}{2} \int j k^{3} a_{k} s_{k} c_{j} c_{k} \partial_{s_{j}} g f d \pi
\end{aligned}
$$

Hence

$$
\begin{aligned}
& \sum_{k \neq j} \frac{1}{2} \int j k\left(s_{j} s_{k} \partial_{c_{j} c_{k}} f+c_{j} c_{k} \partial_{s_{j} s_{k}} f\right) g d \pi-\int j k s_{j} c_{k} \partial_{c_{j} s_{k}} f g d \pi \\
= & \sum_{k \neq j} \frac{1}{2} \int j k\left(s_{j} s_{k} \partial_{c_{j} c_{k}} g+c_{j} c_{k} \partial_{s_{j} s_{k}} g\right) f d \pi-\int j k s_{j} c_{k} \partial_{c_{j} s_{k}} g f d \pi
\end{aligned}
$$

The symmetry of $K$ follows (2.10), (2.11) and (2.16) while the skew-symmetry of $A$ is a consequence of (2.12), (2.13) and (2.14).

As a consequence, we obtain the following Yaglom-reversibility result.

Proposition 2.5. For any smooth functions $f$, we have $G^{*} f(c, s)=J G J f(c, s)$, where $G^{*}$ is the adjoint operator of $G$ in $L^{2}(\pi)$ and $J$ is the operator defined by $J f(c, s)=f(-c,-s)$.

In particular, the time-reversed and flipped process $\left((\tilde{C}(t), \tilde{S}(t))_{t \geq 0}\right.$ has the same distribution as $\left((C(t), S(t))_{t \geq 0}\right.$, where $\tilde{C}(t)=-C(-t)$ and $\tilde{S}(t)=-S(-t)$.

Proof: By Proposition 2.4, it suffices to show that $J K J=K$ and $J A J=-A$. By Definition of $J$, we have $\partial_{c_{j}}(J f)(c, s)=-\left(\partial_{c_{j}} f\right)(-c,-s), \partial_{s_{j}}(J f)(c, s)=$ $-\left(\partial_{s_{j}} f\right)(-c,-s), \quad \partial_{c_{j} c_{k}}(J f)(c, s)=\left(\partial_{c_{j} c_{j}} f\right)(-c,-s), \quad \partial_{s_{j} s_{k}}(J f)(c, s)=$ $\left(\partial_{c_{j} c_{j}} f\right)(-c,-s)$ and $\partial_{c_{j} s_{k}}(J f)(c, s)=\left(\partial_{c_{j} s_{k}} f\right)(-c,-s)$.

Therefore, from (2.8) and (2.9), we get $J K J f=K f$ and $J A J f=-A f$.

We are now in position to prove Theorem 2.1.

Proof of Theorem 2.1:

Throughout the proof, we let $g, h: \mathbb{R}^{n} \rightarrow \mathbb{R}$ denote the functions defined by

$$
g(c, s)=\sum_{k=1}^{n} k a_{k} s_{k} \quad \text { and } \quad h(c, s)=\sum_{k=1}^{n} a_{k} c_{k} .
$$


By Proposition 2.5 and the arguments of Tarrès et al. (2012, Section 3), $B_{t}$ and $\int_{0}^{t} \sum_{k=1}^{n} k a_{k} S_{k}(u) d u$ are uncorrelated. Thus

$$
\begin{aligned}
\mathbb{E}_{\pi}\left(X_{t}^{2}\right) & =t+\mathbb{E}_{\pi}\left(\left(\int_{0}^{t} \sum_{k=1}^{n} k a_{k} S_{k}(u) d u\right)^{2}\right) \\
& =t+2 \int_{0}^{t}(t-u) \mathbb{E}_{\pi}\left(\left(\sum_{k=1}^{n} k a_{k} S_{k}(u) d u\right)\left(\sum_{k=1}^{n} k a_{k} S_{k}(0)\right)\right) d u \\
& =t+2 \int_{0}^{t}(t-u)\left\langle T_{u} g, g\right\rangle_{L^{2}(\pi)} d u
\end{aligned}
$$

By the Cauchy-Schwarz inequality and the third part of Proposition 2.3, $\left\langle T_{u} g, g\right\rangle_{L^{2}(\pi)}$ decreases exponentially fast to 0 . Hence $\int_{0}^{\infty}\left\langle T_{u} g, g\right\rangle_{L^{2}(\pi)} d u$ exists and, therefore, it yields

$$
\lim _{t \rightarrow \infty} \frac{\mathbb{E}_{\pi}\left(X_{t}^{2}\right)}{t}=1+\int_{0}^{\infty}\left\langle T_{u} g, g\right\rangle_{L^{2}(\pi)} d u:=\sigma^{2}
$$

Now that the existence of $\sigma^{2}$ is established, let us prove the bounds in (2.1). The lower bound is trivial since it follows from (2.17). In order to establish the upper bound, we follow the arguments presented in Olla (2001) based on the KipnisVaradhan's CLT theorem.

By Proposition 2.4, we have for any smooth function $f$ having compact support

$$
\langle G f, f\rangle_{L^{2}(\pi)}=\langle K f, f\rangle_{L^{2}(\pi)}=-\frac{1}{2} \int\left(\sum_{j=1}^{n} j\left(s_{j} \partial_{c_{j}} f-c_{j} \partial_{s_{j}} f\right)\right)^{2} d \pi
$$

Hence, using the notation of Olla (2001), we have

$$
\langle G f, f\rangle_{L^{2}(\pi)}=-\|f\|_{1}^{2} .
$$

Because

$$
\begin{aligned}
\int h(c, s)\left(\sum_{j=1} j s_{j} \partial_{c_{j}} f-j c_{j} \partial_{s_{j}} f\right) d \pi & =\int\left(\sum_{j=1}^{n} j s_{j} \partial_{c_{j}} h-j c_{j} \partial_{s_{j}} h\right) f d \pi \\
& =\int g f d \pi
\end{aligned}
$$

it follows from the Cauchy-Schwarz inequality that

$$
\left|\int g f d \pi\right| \leq\|h\|_{L^{2}(\pi)}\|f\|_{1}
$$

Hence, with the notation of Olla (2001),

$$
\|g\|_{-1} \leq\|h\|_{L^{2}(\pi)}=\sqrt{\sum_{j=1}^{n} \frac{a_{j}}{j^{2}}}
$$

Thus, the upper bound comes from Eq. (2.1.7) in Olla (2001).

The second part of the Theorem is immediate due to the third part of Proposition (2.3). 


\section{The two-dimensional case}

The purpose of this section is to provide an extension to the two-dimensional case. More precisely, let $X(t)=\left(X_{1}(t), X_{2}(t)\right)$ be the solution of the self-repelling diffusion

$$
d X(t)=d B(t)-\left(\nabla G(X(t))+\int_{0}^{t} \nabla F(X(t)-X(s)) d s\right) d t,
$$

where $B$ is a two-dimensional standard Brownian motion, $F$ is the interaction potential defined by

$$
F\left(x_{1}, x_{2}\right)=\sum_{k, \ell=1}^{n} a_{\ell, k} \cos \left(\ell x_{1}\right) \cos \left(k x_{2}\right)
$$

and $G$ is the initial potential defined by

$$
\begin{aligned}
G\left(x_{1}, x_{2}\right)= & \sum_{k, \ell=1}^{n}\left(u_{1}^{\ell, k} \cos \left(\ell x_{1}\right) \cos \left(k x_{2}\right)+u_{2}^{\ell, k} \cos \left(\ell x_{1}\right) \sin \left(k x_{2}\right)\right) \\
& +\sum_{k, \ell=1}^{n}\left(u_{3}^{\ell, k} \sin \left(\ell x_{1}\right) \cos \left(k x_{2}\right)+u_{4}^{\ell, k} \sin \left(\ell x_{1}\right) \sin \left(k x_{2}\right)\right) .
\end{aligned}
$$

The result is then the following.

\section{Theorem 3.1.}

Let $\left(X_{t}\right)_{t}$ be the solution of (3.1). Then:

(1) $\sigma^{2}:=\lim _{t \rightarrow \infty} \frac{\mathbb{E}_{\pi}\left(\left|X_{t}\right|^{2}\right)}{t}$ exists

(2) For any $0<t_{1}<\cdots<t_{n}<\infty$, we have

$$
\left(\frac{\sqrt{\varepsilon} X_{t_{1} / \varepsilon}}{\sigma}, \cdots, \frac{\sqrt{\varepsilon} X_{t_{n} / \varepsilon}}{\sigma}\right) \stackrel{\varepsilon \rightarrow 0}{\Longrightarrow}\left(W_{t_{1}}, \cdots, W_{t_{n}}\right)
$$

under $\mathbb{P}_{\pi}$, where $W$ is a two-dimensional standard Brownian motion.

The proof exactly the same way as for the one-dimensional case, though the computations are much more cumbersome. Therefore, we only present the initial steps.

As for the one-dimensional case, we introduce the following variables

$$
\begin{aligned}
& U_{1}^{\ell, k}(t)=u_{1}^{\ell, k}+\int_{0}^{t} \cos \left(\ell X_{1}(s)\right) \cos \left(k X_{2}(s)\right) d s, \\
& U_{2}^{\ell, k}(t)=u_{2}^{\ell, k}+\int_{0}^{t} \cos \left(\ell X_{1}(s)\right) \sin \left(k X_{2}(s)\right) d s, \\
& U_{3}^{\ell, k}(t)=u_{3}^{\ell, k}+\int_{0}^{t} \sin \left(\ell X_{1}(s)\right) \cos \left(k X_{2}(s)\right) d s, \\
& U_{4}^{\ell, k}(t)=u_{4}^{\ell, k}+\int_{0}^{t} \sin \left(\ell X_{1}(s)\right) \sin \left(k X_{2}(s)\right) d s .
\end{aligned}
$$


These variables allow us to extend (3.1) into the following standard stochastic differential equation

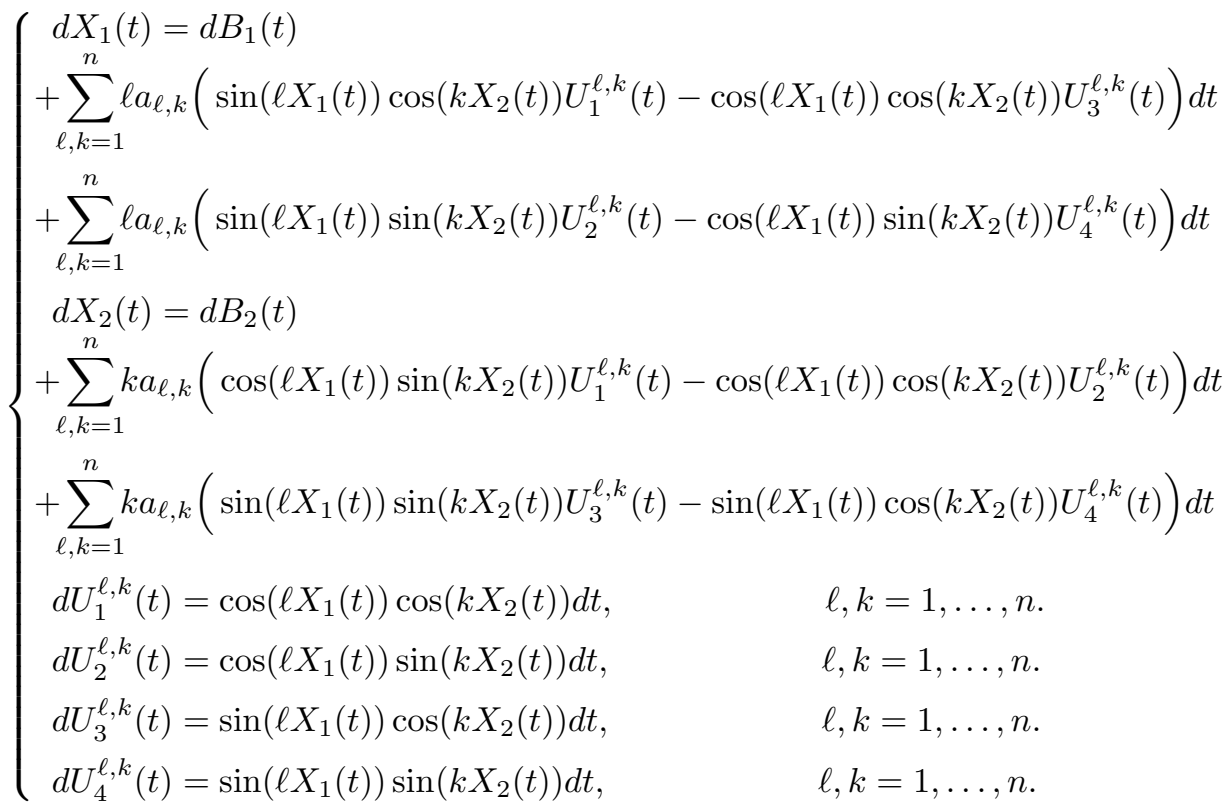

Adopting the point of view of the particle as in Section 2 (see also Horváth et al., 2012) brings us to introduce the following variables.

$$
\begin{aligned}
C_{1}^{\ell, k}(t) & =\cos \left(\ell X_{1}(t)\right) \cos \left(k X_{2}(t)\right) U_{1}^{\ell, k}(t)+\sin \left(\ell X_{1}(t)\right) \cos \left(k X_{2}(t)\right) U_{3}^{\ell, k}(t) \\
& +\cos \left(\ell X_{1}(t)\right) \sin \left(k X_{2}(t)\right) U_{2}^{\ell, k}(t)+\sin \left(\ell X_{1}(t)\right) \sin \left(k X_{2}(t)\right) U_{4}^{\ell, k}(t) \\
S_{1}^{\ell, k}(t) & =\sin \left(\ell X_{1}(t)\right) \cos \left(k X_{2}(t)\right) U_{1}^{\ell, k}(t)-\cos \left(\ell X_{1}(t)\right) \cos \left(k X_{2}(t)\right) U_{3}^{\ell, k}(t) \\
& +\sin \left(\ell X_{1}(t)\right) \sin \left(k X_{2}(t)\right) U_{2}^{\ell, k}(t)-\cos \left(\ell X_{1}(t)\right) \sin \left(k X_{2}(t)\right) U_{4}^{\ell, k}(t) \\
C_{2}^{\ell, k}(t) & =-\sin \left(\ell X_{1}(t)\right) \sin \left(k X_{2}(t)\right) U_{1}^{\ell, k}(t)+\sin \left(\ell X_{1}(t)\right) \cos \left(k X_{2}(t)\right) U_{2}^{\ell, k}(t) \\
& +\cos \left(\ell X_{1}(t)\right) \sin \left(k X_{2}(t)\right) U_{3}^{\ell, k}(t)-\cos \left(\ell X_{1}(t)\right) \cos \left(k X_{2}(t)\right) U_{4}^{\ell, k}(t) \\
S_{2}^{\ell, k}(t) & =\cos \left(\ell X_{1}(t)\right) \sin \left(k X_{2}(t)\right) U_{1}^{\ell, k}(t)-\cos \left(\ell X_{1}(t)\right) \cos \left(k X_{2}(t)\right) U_{2}^{\ell, k}(t) \\
& +\sin \left(\ell X_{1}(t)\right) \sin \left(k X_{2}(t)\right) U_{3}^{\ell, k}(t)-\sin \left(\ell X_{1}(t)\right) \cos \left(k X_{2}(t)\right) U_{4}^{\ell, k}(t) .
\end{aligned}
$$

So, if we denote by $\eta_{t}$ the potential viewed from the particle's position, i.e

$$
\eta_{t}(x)=\int_{0}^{t} F\left(x+X_{t}-X_{s}\right) d s+G\left(x+X_{t}\right)
$$


then

$$
\begin{aligned}
\eta_{t}(x) & \left.=\sum_{\ell, k=1}^{n} a_{\ell, k}\left(\cos \left(\ell x_{1}\right) \cos \left(k x_{2}\right) C_{1}^{\ell, k}(t)-\sin \left(\ell x_{1}\right) \sin \left(k x_{2}\right) C_{2}^{\ell, k}(t)\right)\right) \\
& -\sum_{\ell, k=1}^{n} a_{\ell, k}\left(\sin \left(\ell x_{1}\right) \cos \left(k x_{2}\right) S_{1}^{\ell, k}(t)+\cos \left(\ell x_{1}\right) \sin \left(k x_{2}\right) S_{2}^{\ell, k}(t)\right)
\end{aligned}
$$

and

$$
d X(t)=d B_{t}-\int_{0}^{t} \nabla \eta_{s}(0) d s .
$$

Finally, observe that those new variables solves the following stochastic differential equation

$$
\begin{aligned}
\left(\begin{array}{c}
C_{1}^{\ell, k}(t) \\
S_{1}^{\ell, k}(t) \\
C_{2}^{\ell, k}(t) \\
S_{2}^{\ell, k}(t)
\end{array}\right) & =\ell\left(\begin{array}{c}
-S_{1}^{\ell, k}(t) \\
C_{1}^{\ell, k}(t) \\
-S_{2}^{\ell, k}(t) \\
C_{2}^{\ell, k}(t)
\end{array}\right)\left(d B_{1}(t)+\left(\sum_{p, j=1}^{n} p a_{p, j} S_{1}^{p, j}(t)\right) d t\right) \\
& +k\left(\begin{array}{c}
-S_{2}^{\ell, k}(t) \\
C_{2}^{\ell, k}(t) \\
-S_{1}^{\ell, k}(t) \\
C_{1}^{\ell, k}(t)
\end{array}\right)\left(d B_{2}(t)+\left(\sum_{p, j=1}^{n} j a_{p, j} S_{2}^{p, j}(t)\right) d t\right) \\
& -\frac{\ell^{2}+k^{2}\left(\begin{array}{c}
C_{1}^{\ell, k}(t) \\
S_{1}^{\ell, k}(t) \\
C_{2}^{\ell, k}(t) \\
S_{2}^{\ell, k}(t)
\end{array}\right) d t+\left(\begin{array}{c}
1 \\
0 \\
1 \\
0
\end{array}\right) d t}{2}
\end{aligned}
$$

and that

$$
C_{1}^{\ell, k}(t)^{2}+C_{2}^{\ell, k}(t)^{2}+S_{1}^{\ell, k}(t)^{2}+S_{2}^{\ell, k}(t)^{2}=U_{1}^{\ell, k}(t)^{2}+U_{2}^{\ell, k}(t)^{2}+V_{1}^{\ell, k}(t)^{2}+V_{2}^{\ell, k}(t)^{2} .
$$

Now that the variables describing the environment viewed from the particle's framework are introduced, it suffices the steps from Section 2 since Theorem 2.2 admits a 2-dimensional version. Therefore, we will not repeat them.

\section{Acknowledgement}

I am grateful to Bálint Tóth for asking me whether or not a CLT result holds in the periodic case considered in this work and to the anonymous referee for the useful and constructive comments that have improved the presentation of the paper.

\section{References}

M. Benaïm and C.-E. Gauthier. Self-repelling diffusions on a Riemannian manifold.

Probab. Theory Related Fields 169 (1-2), 63-104 (2017). MR3704766.

I. Horváth, B. Tóth and B. Vető. Diffusive limits for "true" (or myopic) self-avoiding random walks and self-repellent Brownian polymers in $d \geq 3$. Probab. Theory Related Fields 153 (3-4), 691-726 (2012). MR2948690. 
S. Olla. Central limit theorems for tagged particles and for diffusions in random environment. In Milieux aléatoires, volume 12 of Panor. Synthèses, pages 75-100. Soc. Math. France, Paris (2001). MR2226846.

D. Revuz and M. Yor. Continuous martingales and Brownian motion, volume 293 of Grundlehren der Mathematischen Wissenschaften [Fundamental Principles of Mathematical Sciences]. Springer-Verlag, Berlin, third edition (1999). ISBN 3540-64325-7. MR1725357.

P. Tarrès, B. Tóth and B. Valkó. Diffusivity bounds for 1D Brownian polymers. Ann. Probab. 40 (2), 695-713 (2012). MR2952088. 